\title{
Report
}

\section{Thinking about the self influences thinking in general: cognitive consequences of salient self-concept}

\author{
Ulrich Kühnen $^{\mathrm{a}}$ and Daphna Oyserman ${ }^{\mathrm{b}, *}$ \\ ${ }^{\text {a }}$ University of Mannheim, LS Sozialpsychologie, L13,17, D-68131 Mannheim, Germany \\ ${ }^{\mathrm{b}}$ Institute for Social Research, University of Michigan, Ann Arbor, MI 48106-1248, USA
}

Received 20 April 2001; received in revised form 4 December 2001

\begin{abstract}
Two studies support our hypothesis that connected and interdependent self-focus evokes a generally context-dependent cognitive mode (focused on object-context relations) and provide some evidence that separate and independent self-focus evokes a generally context-independent cognitive mode (focused on objects, independent of contexts). Consistent with our predictions, experimental manipulation of interdependent self-focus influences cognitive speed/accuracy (Experiment 1) and memory (Experiment 2). When primed self-focus is congruent with the perceptual task at hand, perceptual speed increases (as shown by a significant task by prime interaction effect) and when primed, interdependent self-focus improves memory for incidentally encoded contextual information. Further research to link primed and chronic self-focus effects is called for. (c) 2002 Elsevier Science (USA). All rights reserved.
\end{abstract}

Keywords: Independence; Interdependence; Self-construal; Priming individualism/collectivism self-focus; Cognition; Memory

Fundamentally, the self is both separate from and connected to others. Yet while everyone can think of the self as separate or as connected, cross-cultural (Markus \& Kitayama, 1991; Rhee, Uleman, Lee, \& Roman, 1995; Trafimow, Triandis, \& Goto, 1991), gender (Markus \& Oyserman, 1989; Cross \& Madson, 1997), and racial-ethnic (Oyserman \& Markus, 1993) research point to between group differences in whether the self is typically construed as related to or separate from others. Terming this difference agency-communion (Bakan, 1966), individualism-collectivism (Gaines et al., 1997) or independence-interdependence (Markus \& Kitayama, 1991), each of these literatures posits chronic stable differences between groups (gender, race, or cultural) in chronically available self-knowledge.

\section{Independent and interdependent self-knowledge: content and process}

However, current social psychological models of the self-concept posit that the self is fundamentally not just

\footnotetext{
${ }^{*}$ Corresponding author.

E-mail addresses: ukuehnen@uni-mannheim.de (U. Kühnen), daphna.oyserman@umich.edu (D. Oyserman).
}

a repository of self-knowledge (Oyserman, 2001). Rather, it is both a set of content-autobiographic memories and semantic information, and a cognitive structure with executive functions that organize information processing (Kühnen, Hannover, \& Schubert, 2001; Markus \& Wurf, 1987; Oyserman \& Markus, 1993). If this argument is true then researchers should be able to see the impact of self-concept content on cognitive processing style. In the case of independent vs. interdependent self-knowledge, developing and maintaining an independent self-view would involve a different cognitive processing style than developing and maintaining an interdependent self-view. Specifically, seeing one's self as independent of others, contexts and situations requires a context-independent processing style-aggregating and integrating across situations, ignoring situational variance in one's thoughts, feelings, and responses. Seeing one's self as interdependent with others, contexts, and situations requires a context-dependent processing style-paying attention to specific social contexts. What is the evidence that independent and interdependent self-construals influence judgment and cognitive processing either chronically or when temporarily made salient? 


\section{Indirect evidence}

With few exceptions, evidence is indirect-based on between-group comparisons that assume rather than measure posited differences in interdependence-independence. For example, reasoning from the literature on gender and national differences in independence-interdependence, authors assume that people of different genders or nationalities should differ in spontaneous judgments (with interdependence associated with assimilating social comparison information and independence associated with contrasting the self to comparison standards). This is a plausible hypothesis with some support, women, not men, assimilate an other's failure into their self-assessment, feeling more pessimistic about their chances of success after bringing to mind the other (Kemmelmeier \& Oyserman, 2001a). Koreans, not Americans, consider salient contextual information when trying to explain an outcome (Choi \& Nisbett, 1998). In these and other studies (e.g., Heine \& Lehman, 1995), between-group differences in judgment were attributed to between group differences in self-views. Indeed indirect evidence is more focused on the linkage between interdependent self-views and contextualized reasoning than the reverse-independent self-views and decontextualized reasoning.

\section{Direct evidence}

Relating self-knowledge to semantically similar judgments. Though plausible, gaps in previous research limit the causal claims that can be made. First, as noted above, most previous research does not measure or manipulate independence-interdependence but rather infers it from group membership so that causal role of self-construals cannot be examined directly. Cognitive priming allows researchers to test the causal consequences of accessible self-knowledge directly. Because individuals use accessible knowledge to make sense of the judgment tasks at hand, primed (experimentally manipulated) independent or interdependent self-knowledge is more likely to influence judgment than is other self-knowledge that is not made salient (Bargh, Chen, \& Burrows, 1996; Higgins, Rholes, \& Jones, 1977; Srull \& Wyer, 1979).

Even when independent vs. interdependent self-construal content is measured (e.g., Oyserman, Sakamoto, \& Lauffer, 1998) or its salience manipulated (e.g., Gardner, Gabriel, \& Lee, 1999; Kemmelmeier \& Oyserman, 2001b; Kühnen \& Hannover, 2000; Trafimow et al., 1991), previous research typically uses as dependent variables social judgments semantically linked to independence-interdependence-social obligation, relationship closeness, feelings of optimism about the future (see Oyserman, Coon, \& Kemmelmeier, 2002a for a review). Given the semantic link between the content of independent (interdependent) self-construals and the content of the dependent variables, current research cannot shed light on whether results are due to activation of semantically linked relevant knowledge or if in fact selfconstrual differences carry with them different cognitive processes (modes of thinking).

Relating self-knowledge to processing style. In a first test of the influence of independent and interdependent self-knowledge on processing style, Kühnen et al. (2001) examined the effect of priming independent vs. interdependent self-knowledge (using Gardner and colleague's (1999) prime) on performance on two tasks, an adapted version of the Embedded-Figures-Test (Witkin, Oltman, Raskin, \& Karp, 1971) and a second picture completion task. They showed that independence-primed participants solved more of the embedded-figure problems in a given time while interdependence-primed participants solved more of the incomplete pictures. The embeddedfigures task requires finding simple pictures within complex ones and the picture completion task requires finding what is missing in the presented complex contexts. The embedded-figures task seemed to be facilitated by independent self-focus while the picture completion task, seemed to be facilitated by interdependent self-focus.

While this research provides initial evidence supporting the assumption that self-concept focus actually shifts cognitive processing style, further replication is important for a number of reasons. It is important first, because the tasks differed in the extent that they were free of semantic content. The picture-completion task utilized stimuli that might be considered semantically related to the priming (for example one picture required that participants notice that in a picture including sun, a tree, and a man, the tree had a shadow, but the man did not). To the extent that interdependence carries with a focus on the social, it might be that this prime influenced content rather than process. Second, the measures were of outcomes (correct responses), so a more process-oriented measure would be helpful. Third, the studies relied on between-subjects effects. A manipulation that showed within subject effects would underscore the robustness of the effect of temporary salience of self-concept content on cognitive processing style.

Current research. The presented experiments provide conceptual replication and extension of the few available previous experimental findings. First, given the very different nature of the cognitive tasks used by Kühnen et al. (2001) further evidence for the validity of the assumption that self-construal priming affects participants' processing styles is required. Second, since our hypotheses predict procedural consequences of selfconstrual priming, we developed a more process oriented, online-measurement of context-dependency in information processing. Third, the studies by Kühnen et al. (2001) are limited in that these authors provided cognitive tasks requiring either context-independent or context-dependent processing to different participants. 
Therefore, in Experiment 1 we measured within subjects both context-dependent and context-independent processing after independent-interdependence priming. A fourth limitation of the Kühnen et al. (2001) studies is that they investigated solely perceptual consequences of self-construal activation. If perception is influenced by self-construal priming, then one might expect that memory performance for the perceived (contextualized vs. non-contextualized) objects is likely to be influenced as well. Experiment 2 therefore extends the research to memory performance.

\section{Experiment 1}

Experiment 1 conceptually replicates previous findings using: (a) different materials, (b) a different dependent measure-speed of (accurate) processing rather than number of correct responses, and (c) a within subjects design in which participants responded to two different cognitive tasks, one requiring context-independent thinking and the other requiring context-dependent thinking.

\section{Method}

\section{Participants}

Thirty undergraduates, randomly assigned to priming condition, participated for course credit. Race/ethnicity was obtained by linking student id to response on a previous, unconnected, questionnaire filled out in the first weeks of the semester. Most participants were white $(n=14)$, other descriptions were "black" 1, "East Asian" 3, "South Asian" 3, "Hispanic" 1, "bi-racial" 1 ,"none of the above" 1 , and 6 participants did not indicate their ethnicity.

\section{Materials}

We primed independent vs. interdependent selfknowledge using Gardner and colleagues' (1999) pronoun circling manipulation, in which participants read a brief paragraph about a trip to a city, and are instructed to circle the 19 pronouns in the text. In the independence priming condition, the pronouns represented the individual self (e.g., "I," "me," "mine"), while in the interdependence priming condition the pronouns represented the relational self (e.g., "we," "our," "us"). Gardner et al. (1999) showed that these primes elicited values and attitudes congruent with the primed way of thinking about one's self.

To measure context-dependency in information processing, we constructed a letter identification task. Participants saw a letter made up of smaller letters on a computer screen, below the stimulus letter were two response options, labeled ' 1 ' and '2.' Fig. 1 displays an example screen, in this example, small F's form an $\mathrm{H}$

$\begin{array}{lr}\text { F } & \text { F } \\ \text { F } & \text { F } \\ \text { F } & \text { F } \\ \text { FFFFFFF } \\ \text { F } & \text { F } \\ \text { F } & \text { F } \\ \text { F } & \text { F }\end{array}$

Fig. 1. Letter task stimulus.

(font size 18), and the response options corresponding to 1 and 2 are $\mathrm{H}$ and $\mathrm{F}$, respectively. We chose this task because either a context-dependent or context-independent cognitive processing style would be beneficial, depending on whether the task was to find the large letter or find the small letter: Identifying the small letters requires focussing on the elements, while ignoring the large letter (i.e., the context) they form. Identifying the large letters requires focusing on the context, the relationships among the elements.

Procedure. Half of the participants were primed with the independent prime and half with the interdependent prime. In each priming condition, half of the participants were first asked to identify the small letter ( $\mathrm{F}$ in our example) presented on the screen as rapidly as possible and half were first asked to identify the large letter presented on the screen as rapidly as possible $(\mathrm{H}$ in our example). The correct response was randomly assigned to the ' 1 ' or the ' 2 ' key. Participants were to press the key corresponding to their choice as fast as possible. Stimuli remained on the screen until participants identified the letter. Each trial block included 36 stimuli. After completing the first trial block, participants were re-primed with the same self-focus as was primed prior to the first trial block. Whereas the first self-focus priming task was exactly the Gardner et al. (1999) pronoun task (1999), the re-priming task was in the same form and used the same number and type of pronouns but instead of the paragraph describing a day in the city, it described a day on the farm. In the second trial block, participants received the same 36 stimuli as in the first trial block. This time, however, the to-beidentified letters were the opposite of those identified in the first block: That is, if participants had identified the large letters in the first trial block, they were now asked to identify the small letters, and vice versa. Thus, the letter identification task (small or large letter) varied within participants, and the priming condition (independence or interdependence) and the order of the tasks (small or large letter first) varied between participants. 
Participants were debriefed, thanked, and dismissed after the second letter task.

\section{Results}

We expected that the independence prime would facilitate rapid identification of the small letters (decontextualized processing) relative to identification of the large letters (contextualized processing), and that reverse would be found in interdependence-primed participants. Given the within subjects design, this hypothesis would lead to a crossover interaction in which priming would speed up processing in the congruently focused task relative to the incongruently focused task. To test our hypothesis, the latencies of correct responses were first log-transformed and then averaged for each task (large letter and small letter). ${ }^{1}$ The log-transformed response latency scores were submitted to a 2 (priming: independence vs. interdependence) $\times 2$ (order of tasks: large vs. small letter first) $\times 2$ (task: large vs. small letter identification) ANOVA. The first two factors were varied between, the latter one within participants. As predicted, we found a significant interaction effect of priming and task; $F(1,28)=4.28 ; p=.048$. The pattern of results was in line with our predictions. Independence primed participants were quicker in identifying the small $(M=2.908)$ as compared to the large letters $(M=2.948$; $t(14)=2.01 ; p=.03$ ), while the opposite pattern (although not significant) was found for interdependence primed participants $(M=2.904$ vs. $M=2.89 ; t(14)=$ $-.83 ; p=.21)$. Fig. 2 presents the mean untransformed response latencies in each condition.

We expected that primed processing style would influence processing speed, speeding up congruent relative to incongruent processing. Thus the effect critical to test our hypothesis was a crossover interaction, which we found. Moreover, the simple effect for independence self-focus priming was significant; and though the simple effect for interdependence self-focus was not significant, it was in the right direction. We did not expect and did not find a main effect of task or of prime (both $F_{\mathrm{s}}<1$ ). Although not part of the initial design, we explored whether priming might also influence other aspects of cognitive processing (e.g., error rate) and whether

\footnotetext{
${ }^{1}$ Effects were robust. Effects did not differ when all responses (accurate and erroneous) rather than only correct responses were included in the response-latency measure. Recall that order of letter task was balanced such that half of respondents did the large letter task first and half did the small letter task first. This proved important since we did find a significant practice effect, a 2-way interaction of task type and task order on mean response latency (using a 2 (tasks) $\times 2$ (priming) $\times 2$ (order of tasks) ANOVA), $F(1,26)=11.28 ; p=.002$, such that the second task was completed more quickly than the first. If identifying large letters was done as the second task, identification was faster $(M=2.89)$ than if this task was done first $(M=2.94)$ and if identifying small letters was done as the second task, identification was faster $(M=2.89)$ than if this task was done first $(M=2.92)$.
}

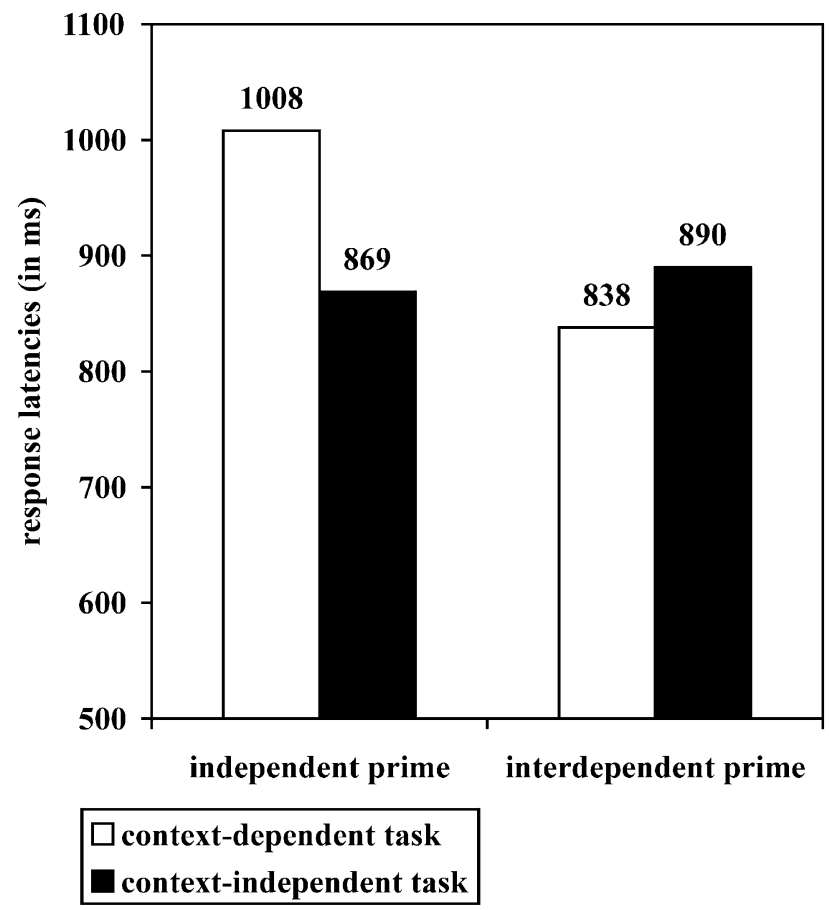

Fig. 2. Mean untransformed response latencies.

chronic differences in self-focus might interact with primed differences in self-focus to influence cognitive processing. Since we chose such a simple experimental task, a differential error rate by condition was not expected. Indeed, error rate was extremely low (mean number of errors overall conditions was .31; i.e., an error ratio of $0.43 \%$ ) and did not differ by experimental condition (all $F \mathrm{~s}<1)$. However, in a more complex task, error rate rather than response time might be an important measure. To explore possible interaction between primed and chronic self-focus, we introduced participants' gender as a next step of analysis in the ANOVA design described above. Gender did not have any significant main or interaction effect, suggesting that the self-focus we primed rather than self-focus that was chronically accessible was driving the self-focus prime by task interaction effect. Although chronic difference in self-focus might also be studied by using race or ethnic background, our sample was not adequate to test for stable cross-cultural differences in self-construal since only seven participants were Asian, East Asian, or Hispanic (groups found significantly different from White Americans on chronic interdependent self-focus, see Oyserman et al., 2002a). However, it is possible that chronic self-focus may interact with primed self-focus and task to create a three-way interaction effect.

\section{Discussion}

The expected prime by task interaction was significant and the overall pattern of means was in the predicted 
direction-mean processing speed of decontextualized information was faster than mean processing speed of contextualized information for independent self-construal primed individuals and the reverse tended (nonsignificantly) to be the case for interdependence primed individuals. Independent self-knowledge primed participants saw the component small letters faster than they saw the large letter made up of these smaller letters, while the opposite tendency was observed in participants primed with interdependent self-knowledge. To our knowledge, this is the first demonstration that self-construal priming affects modes of thinking, while varying within participants the nature of the provided cognitive tasks requiring either context-independent or contextdependent processing. Our findings support the assumption that independent and interdependent self-knowledge foster different modes of thinking. Independent self-knowledge is associated with a context-independent mode of processing, while interdependent self-knowledge is less so. Note that our findings focus on the relative effects of priming. We did not use a nonprimed control group because it would be unclear what the unprimed participants were focused on and subtle features of the experimental situation may have focused their attention one way or another such that no true 'control' is possible. Our argument has to do with speed of processing with one self-focus relative to another, rather than in relation to some theoretically "non-focused' condition. However, in future studies, a no prime control group might be more effective for detecting chronic self-focus influences, like gender-based or ethnicbased differences.

The careful reader also will note that the difference between the priming conditions was much more pronounced on the large than on the small letter task. Whereas on the large letter task, interdependence primed participants were quicker than independence primed participants, no such difference was observed on the small letter task. One possible explanation for this finding is that identifying small letters in the context of many other letters is simply what we naturally do when we read something in everyday life. Therefore, because the task of identifying small letters is more dominant in everyday life, this task may be less diagnostic for the assumed processes.

We used the letter task as a dependent variable, because of its face validity for measuring context-dependent vs. context-independent perception. We later found that other researchers had used similar materials to study other issues. Most relevant, Navon (1997) created similar materials to show that global perception precedes local perception ("seeing the forest before tress," (p. 353)). Specifically he showed that embedding small letters in a large letter slowed small letter recognition more than it slowed recognizing the large letter. His findings reinforce the notion that the small letter task may be an over learned task and therefore less sensitive to priming manipulations of the sort we used.

\section{Experiment 2}

Experiment 1 gave evidence for the assumption that self-construal priming affects the degree of context-dependency in perceptual processes. Experiment 2 was conducted to extend this research into the field of memory processes. In particular, we assumed that if interdependent self-knowledge fosters perceptual processing of objects as related to contexts then memory for incidentally encoded contextual information should improve if interdependent (rather then independent) self-knowledge is accessible. Specifically, we adopted material designed by Chalfonte and Johnson (1996). They argued that memory of complex events requires not only memory of particular features (e.g., item, location, color, etc.), but also cognitive processes for binding these features together. "Binding provides the memorial experience that certain features belong together," (Chalfonte \& Johnson, p. 403). In their research they showed age-related decline in this binding process. Since we assume that interdependent self-knowledge fosters binding objects to the context, we expected interdependence primed participants to outperform independence primed ones on memory tasks requiring such binding.

\section{Method}

\section{Participants}

Thirty-four undergraduate students randomly assigned to priming condition (independence, interdependence salient), obtained course credit for participation. Race/ethnicity was obtained by linking student id to response on a previous, unconnected, questionnaire filled out in the first weeks of the semester. Seventeen participants indicated "White," 1 "Black," 1 "Hispanic," 1 "mixed," 6 "Asian," 1 "none of the above" as their ethnicity, and 7 participants did not indicate their ethnicity at all.

\section{Materials}

We primed independent vs. interdependent selfknowledge using Gardner and colleagues' (1999) pronoun circling manipulation, in which participants read a brief paragraph about a trip to a city, and are instructed to circle the 19 pronouns in the text. In the independence priming condition, the pronouns represented the individual self (e.g., "I", "me," "mine"), while in the interdependence priming condition the pronouns represented the relational self (e.g., "we," "our," "us").

The memory task was modified from Chalfonte and Johnson (1996). Materials were: (a) a $20 \times 12 \mathrm{~cm}$ picture 


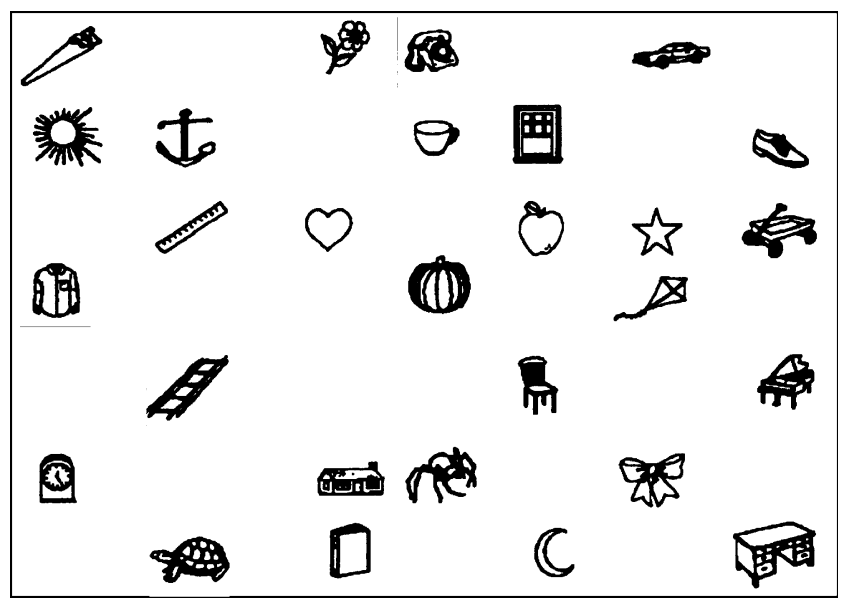

Fig. 3. Study array.

made up of 28 simple items (e.g., house, moon, and train track) without any systematic spatial order presented for $90 \mathrm{~s}$ (see Fig. 3); and (b) an empty grid on which the participant subsequently documented what he or she remembered.

Procedure. Participants were told that they would be involved in two tasks, a pronoun recognition task and a memory task. Participants worked alone. They were told their job would be to circle all pronouns in a text and then take a memory test. After viewing a picture for $90 \mathrm{~s}$, they would need to remember what they had just seen.

The experimenter presented the picture for $90 \mathrm{~s}$ then removed it and provided the participant with a samesized but empty grid sheet. Instructions were "Now I would like you to remember what you have just seen. Please, try to remember what you have seen and where you have seen it. Write down in the cells of this grid the items you saw in the place you saw each one. If you can remember an item, but not where it was, you can write it down outside the grid. Please, try to remember as many items and their positions as possible." These instructions allowed us to code both participants' contextualized memory performance for situated items and their total recall of items, irrespective of their position.

\section{Results}

We predicted that interdependence primed participants would spontaneously process the presented stimuli as contextually situated and thus perform better at the memory task than independence primed individuals. As expected, compared to independence primed participants $(M=6.35 ; S D=3.48)$, interdependence primed participants were better able to remember what they had seen and the location they had seen it in $(M=8.71$, $S D=3.37), \quad t(32)=-2.0 ; \quad p=.03$. Interdependence primed participants outperformed independence primed ones in recalling items plus their location, indicating that they spontaneously encoded the items in context. It was in remembering where items were that interdependence priming produced a clear advantage. When we simply counted number of remembered items, both groups did quite well, (independence primed, $M=14.71 ; S D=$ 3.42 ; interdependence primed, $M=16.0 ; S D=4.0$, $t(32)=-1.01 ; p>.10)$. Since participants did not know that they would subsequently have to remember both items and their positions, their later memory performance is as an indicator of spontaneous binding of object to a place or context.

\section{Discussion}

Our goal was to show that interdependent selfconstruals induce what we called 'context-dependent thinking,' a style of thinking in which information is processed contextually. This context bound processing can be characterized as paying attention to connections among items and the relationship between objects and the field they are presented in. If this is the case, then memory performance for incidentally encoded contextual information (i.e., location) should be influenced. As expected, interdependence-primed participants outperformed independence-primed participants on the contextualized memory task since this is a task in which context-dependent thinking is beneficial. These results, together with the results from previous research (Gardner et al., 1999; Kühnen et al., 2001), provide evidence that when primed, independent and interdependent self-construals influence not only how we think about ourselves, but also how we think - the cognitive processes that are automatically instantiated.

The lack of difference in memory when contextual information is ignored further substantiates our interpretation of the results as based in different thinking styles cued by self-construal focus. Interdependent selfconstrual primed participants did not simply remember more than independence primed participants, thus the results are not simply due to one group of participants slacking off or not trying. Rather, priming interdependent self-construal increased participants' ability to remember contextual information about the items, that is, the location of the item on the field. This effect cannot be interpreted as semantic priming, because there is no semantic relation between the primed self-knowledge and the to be remembered information.

\section{General discussion}

A growing literature suggests chronic cultural differences in cognitive processing - contrasting European American with Chinese or Korean participants to show that these East Asian participants are more likely to process information in terms of the whole or context within which it is presented (e.g., Morris, Nisbett, \& 
Peng, 1995). Building on a broad social-psychological research base showing that the 'on-line' or 'working' self-concept is easily manipulated by subtle priming manipulations (for a review see Baumeister, 1998; Oyserman, 2001), experimental manipulations of self-focus (independence vs. interdependence) have been used to begin to understand the process by which these chronic differences may occur. Previous research showing effects of self-focus on cognition has focused on social cognition, leaving ambiguity as to whether the effect of selffocus was to activate semantically similar information and thus influence cognition or if the effect was related to a more basic shift in processing mode. We argue that the latter is the case.

Using an online-processing measure (Experiment 1) and a memory measure (Experiment 2) of the degree of context-dependency in information processing, the experiments we describe allow stronger conclusions about the effects of self-focus (independence vs. interdependence) on cognition than previous research. We found the predicted influence of self-focus on cognitive style, as measured by processing speed and memory. With regard to processing speed, the overall interaction effect and the effect of priming independence were each significantwithin subjects, priming independent self-focus improved processing speed for decontextualized as compared with contextualized processing, the reverse was true for interdependence priming (though not significantly). With regard to memory, we found that interdependenceprimed participants outperformed independence-primed participants on a memory task requiring context-dependent processing.

Note that priming interdependence (or independence) did not simply prime semantically relevant content like 'togetherness' (or 'uniqueness'); it primed participants to think contextually (or de-contextually). There was no semantic relation between the primed self-knowledge and the dependent variables in either study. Therefore, the present research extends previous findings about the contextual nature of self-construal. It underscores that the implications of increasing interdependent selfconstruals' accessibility are not limited to priming relevant social identities, values, and norms. Together these studies provide evidence that both independent and interdependent self-construals influence information processing and foster opposing modes of thinking with regard to the degree of context-dependency. While the previous literature (Kühnen et al., 2001) has primarily focused on the benefits of independent self-knowledge, the present studies provide evidence of increased performance on context-dependency requiring tasks due to primed interdependent self-knowledge.

Although this research is not focused on chronic differences in self-focus, we believe that our findings can be linked to cross-cultural and gender research. Our findings suggest that when the interdependent-self is chronically 'on line' or available, individuals will have better item-by-context linked perception and memory. Thus, members of collectivist cultures should exhibit better memory performance for such tasks than will members of individualist cultures. This speculation is in line with work by Nisbett and his colleagues, who distinguish the analytic way of processing information by Western culture members from the more holistic system of thought of Chinese participants (see Nisbett, Choi, Peng, \& Norenzayan, 2000 for a review). Our findings provide process-oriented support for the notion that independent and interdependent self-construals influence psychological experience more generally (Kühnen et al., 2001; Markus \& Kitayama, 1991; Oyserman \& Markus, 1993). Our results are also congruent with a growing literature in cultural psychology implicating social contexts in making certain ways of defining the self chronically salient (e.g., Oyserman et al., 2002a, 2002b; Triandis, 1995). Our results add to research reconnecting cultural and gender differences with social psychological models of self-concept (e.g., Cross \& Madson, 1997). Finally, our results provide support for the notion that priming independent and interdependent self-knowledge influences ongoing information processing by inducing the associated cognitive procedures or modes of thinking and not simply by activating socialrelational information about the self.

Because semantic and procedural information are likely to be cued simultaneously, research that simply compares groups differing in gender or culture cannot disentangle these effects. Efforts to understand the implications of cultural and other group differences by describing chronic differences in average levels of independent or interdependent self-construals are similarly limited in their ability to make sense of the process by which influence occurs. To the extent that culture can be understood as a chronic source of activation of different aspects of self-knowledge (e.g., Gardner et al., 1999; Hannover, 1997; Kühnen, 1999; Kühnen \& Hannover, 2000; Oyserman et al., 2002a; Oyserman, Kemmelmeier, \& Coon, 2002b; Trafimow, Silverman, Mei-Tai Fan, \& Shui Fun Law, 1991, 1997), then efforts such as ours which manipulate which self-knowledge is temporarily activated can provide insight into these otherwise elusive processes.

A final note with regard to the limitations of our current studies is warranted. Although we attempted to find a pair of experimental tasks that were parallel in type with one demanding decontextualized (find the small letter) and the other demanding contextualized (find the large letter) processing, our decontextualized task may have been generally simpler since it is so similar to the over learned task of reading. For this reason, the contextualized processing task may well be the more diagnostic of the two dependent variables in Experiment 1. Similarly, in Experiment 2, the dependent 
measure also involved contextualized cognition, in this case, memory. Thus, our research shows the beneficial effects of interdependent self-focus on contextualized reasoning tasks more than the reverse (benefits of independent self-focus on decontextualized reasoning tasks).

\section{Acknowledgments}

The research was supported by the Deutsche Forschungsgemeinschaft (Grant $\mathrm{Ku}$ 1305/1-1 to Kühnen) and the W.T. Grant Foundation (Faculty Scholar Award to Oyserman). I am grateful to the Center for Advanced Studies in the Behavioral Sciences for support during the writing process (Oyserman).

\section{References}

Bakan, D. (1966). The duality of human existence. Boston, MA: Beacon Press.

Bargh, J. A., Chen, M., \& Burrows, L. (1996). Automaticity of social behavior: direct effects of trait construct and stereotype activation on action. Journal of Personality and Social Psychology, 71, 230244.

Baumeister, R. (1998). The self. In D. Gilbert, S. Fiske, \& G. Lindzey (Eds.), Handbook of social psychology (pp. 680-740). New York: Oxford University Press.

Chalfonte, B. L., \& Johnson, M. K. (1996). Feature memory and binding in young and older adults. Memory and Cognition, 24, 403416.

Choi, I., \& Nisbett, R. E. (1998). Situational salience and cultural differences in the correspondence bias and the actor-observer bias. Personality and Social Psychology Bulletin, 24, 949-960.

Coon, H., \& Kemmelmeier, M. (2001). Cultural orientations in the United States: (Re)-examining differences among ethnic/racial groups. Journal of Cross Cultural Psychology, 32, 348-364.

Cross, S., \& Madson, L. (1997). Models of the self: self-construals and gender. Psychological Bulletin, 122, 5-37.

Gaines, S. O., Marelich, W. D., Bledsoe, K. L., Steers, W. N., Henderson, M. C., Granrose, C. S., Barajas, L. H. D., Lyde, M., Takahashi, Y., Yum, N., Rios, D. I., Garcia, B. F., Farris, K. R., \& Page, M. S. (1997). Links between race/ethnicity and cultural values as mediated by racial/ethnic identity and moderated by gender. Journal of Personality and Social Psychology, 72, 14601476.

Gardner, W., Gabriel, S., \& Lee, A. (1999). "I" value freedom but "we" value relationships: self-construal priming mirrors cultural differences in judgment. Psychological Science, 10(4), 321-326.

Hannover, B. (1997). Das dynamische Selbst. Zur Kontextabhängigkeit selbstbezogenen Wissens (The dynamic self. The context-dependency of self-related knowledge). Bern, Switzerland: Huber.

Heine, S. J., \& Lehman, D. R. (1995). Cultural variation in unrealistic optimism: does the West feel more vulnerable than the East? Journal of Personality and Social Psychology, 68, 595-607.

Higgins, E. T., Rholes, W. S., \& Jones, C. R. (1977). Category accessibility and impression formation. Journal of Experimental Social Psychology, 13, 141-154.

Kemmelmeier, M., \& Oyserman, D. (2001a). Gendered influence of downward social comparisons on current and possible selves. Journal of Social Issues, 57, 129-148.

Kemmelmeier, M., \& Oyserman, D. (2001b). Self-construals and consequences of upward social comparison: the ups and downs of thinking about a successful other. European Journal of Social Psychology, 31, 311-320.

Kühnen, U. (1999). Verzerrungen bei direkten und indirekten selbstbezogenen Ähnlichkeitsurteilen als Konsequenz hoch zugänglichen Selbstwissens (Biases in direct and indirect self-other similarity judgments as a consequence of highly accessible self-knowledge). Lengerich, Germany: Pabst Science Publishers.

Kühnen, U., \& Hannover, B. (2000). Assimilation and contrast in social comparisons as a consequence of self-construal activation. European Journal of Social Psychology, 30, 799-811.

Kühnen, U., Hannover, B., \& Schubert, B. (2001). The semanticprocedural-interface model of the self: the role of self-knowledge for context-dependent versus context-independent modes of thinking. Journal of Personality and Social Psychology, 80, 397-409.

Markus, H., \& Kitayama, S. (1991). Culture and the self: Implications for cognition, emotion, and motivation. Psychological Review, 98, 224-253.

Markus, H. R., \& Oyserman, D. (1989). Gender and thought: the role of the self. In M. Crawford, \& M. Hamilton (Eds.), Gender and thought (pp. 100-127). New York: Springer.

Markus, H., \& Wurf, E. (1987). The dynamic self-concept: a social psychological perspective. Annual Review of psychology, 38, 299337.

Morris, M. W., Nisbett, R. E., \& Peng, K. (1995). Causal attribution across domains and cultures. In D. Sperber, D. Premack, \& A. J. Premack (Eds.), Causal cognition: A multidisciplinary debate (pp. 577-613). Oxford: Oxford University Press.

Navon, D. (1997). Forest before trees: the precedence of global features in visual perception. Cognitive Psychology, 9, 353-383.

Nisbett, R. E., Choi, I., Peng, \& Norenzayan, A. (2000). Culture and systems of thought: Holistic vs. analytic cognition. Ann Arbor, MI: The University of Michigan.

Oyserman, D. (2001). Self-concept and identity, 2001. In A. Tesser, \& N. Schwarz (Eds.), The Blackwell Handbook of Social Psychology (Vol. I) (pp. 402-415). Malden, Mass: Blackwell Publishers.

Oyserman, D., Coon, H., \& Kemmelmeier, M. (2002a). Rethinking individualism and collectivism: Evaluation of theoretical assumptions and meta-analyses. Psychological Bulletin, 128, 3-72.

Oyserman, D., Kemmelmeier, M., \& Coon, H. (2002b). Cultural psychology: A new look. sychological Bulletin, 128, 110-117.

Oyserman, D., \& Markus, H. R. (1993). The sociocultural self. In J. Suls (Ed.), Psychological perspectives on the self (Vol. 4) (pp. 187220). Hillsdale, NJ: Erlbaum.

Oyserman, D., Sakamoto, I., \& Lauffer, A. (1998). Cultural accomodation: hybridity and the framing of social obligation. Journal of Personality and Social Psychology, 74, 1606-1618.

Rhee, E., Uleman, J. S., Lee, H. K., \& Roman, R. J. (1995). Spontaneous self-descriptions and ethnic identities in individualistic and collectivistic cultures. Journal of Personality and Social Psychology, 69, 142-152.

Srull, T. K., \& Wyer, R. S. (1979). The role of category accessibility in the interpretation of information about persons: some determinants and implications. Journal of Personality and Social Psychology, 37, 1660-1672.

Trafimow, D., Triandis, H. C., \& Goto, S. G. (1991). Some tests of the distinction between the private self and the collective self. Journal of Personality and Social Psychology, 60, 649-655.

Trafimow, D., Silverman, E. S., Mei-Tai Fan, R., \& Shui Fun Law, J. (1997). The effects of language and priming on the relative accessibility of the private and the collective self. Journal of Cross-Cultural Psychology, 28, 107-123.

Triandis, H. (1995). Individualism \& collectivism. Boulder, CO, US: Westview Press.

Witkin, H. A., Oltman, P. K., Raskin, E., \& Karp, S. A. (1971). Manual for the Embedded Figures Test, Children's Embedded Figures Test, and Group Embedded Figures Test. Palo Alto, CA: Consulting Psychologists Press. 